\title{
Reliability Modeling and Optimization Strategy for Manufacturing System Based on RQR Chain
}

\author{
Yihai He, ${ }^{1,2}$ Zhenzhen He, $^{1}$ Linbo Wang, and Changchao Gu ${ }^{1}$ \\ ${ }^{1}$ School of Reliability and Systems Engineering, Beihang University, Beijing 100191, China \\ ${ }^{2}$ Department of Systems Engineering and Engineering Management, City University of Hong Kong, Kowloon, Hong Kong \\ Correspondence should be addressed to Yihai He; hyh@buaa.edu.cn
}

Received 12 June 2015; Accepted 2 November 2015

Academic Editor: Nidhal Rezg

Copyright (c) 2015 Yihai He et al. This is an open access article distributed under the Creative Commons Attribution License, which permits unrestricted use, distribution, and reproduction in any medium, provided the original work is properly cited.

\begin{abstract}
Accurate and dynamic reliability modeling for the running manufacturing system is the prerequisite to implement preventive maintenance. However, existing studies could not output the reliability value in real time because their abandonment of the quality inspection data originated in the operation process of manufacturing system. Therefore, this paper presents an approach to model the manufacturing system reliability dynamically based on their operation data of process quality and output data of product reliability. Firstly, on the basis of importance explanation of the quality variations in manufacturing process as the linkage for the manufacturing system reliability and product inherent reliability, the RQR chain which could represent the relationships between them is put forward, and the product qualified probability is proposed to quantify the impacts of quality variation in manufacturing process on the reliability of manufacturing system further. Secondly, the impact of qualified probability on the product inherent reliability is expounded, and the modeling approach of manufacturing system reliability based on the qualified probability is presented. Thirdly, the preventive maintenance optimization strategy for manufacturing system driven by the loss of manufacturing quality variation is proposed. Finally, the validity of the proposed approach is verified by the reliability analysis and optimization example of engine cover manufacturing system.
\end{abstract}

\section{Introduction}

To meet the demands of high reliability and long life of the product, integrated analysis, assurance, and optimization for reliability are required to be carried out in the lifecycle of design, manufacture, and usage. However, for a long time, most of traditional reliability studies had merely focused on the design and usage stages, and reliability technology suitable for the manufacturing process has always been ignored, lacking proper attention it deserved, which caused the serious degradation of product reliability after batch production frequently, and resulting in a high infant failure rate, and the product inherent reliability could not meet the increasingly stringent design reliability requirements [1]. As we all know, product is the output of the manufacturing process which is the implementation form of the manufacturing system. Therefore, the reliability of final produced product is closely related to the reliability of manufacturing system and the quality of manufacturing process. Usually, even a good design cannot guarantee that the manufactured products achieve the satisfactory reliability when the design quality of manufacturing system is poor [2]. Thus, it can be seen that integrating the reliability modeling and optimization for manufacturing system is crucial to ensure the product reliability.

Practices have proved that uncertain factors like quality variation in manufacturing process and deteriorations of system components could lead to the degradation of manufacturing system, which should affect the quality of manufacturing process interactively. And when quality variations are cumulated and amplified, the number of potential defects of products is arising, which would trigger the decline of product inherent reliability finally. In order to minimize the decline of product inherent reliability with respect to the design specifications, identifying and optimizing the critical factors in manufacturing that contribute to the product reliability degradation systematically are becoming the research focus of reliability engineering currently, and how 
to carry out product reliability oriented reliability modeling and optimization of manufacturing system is the most urgent and task.

At different nodes of product life cycle, product reliability exhibits different characteristics. Murthy [3] defined the evolution chain which transfers product reliability from design, manufacturing, transportation, sale, and usage, enriching notation of product reliability at different stages, and named the reliability in manufacturing as product inherent reliability. Then, Jiang and Murthy [4] pointed out the negative impact of variations on the reliability during the product life cycle via the transmission chain and pointed out that the quality variations and assembly errors are the root reasons causing the deterioration of product inherent reliability. As to product inherent reliability in manufacturing, $\mathrm{Li}$ et al. $[5,6]$ noted that both the reliability of manufacturing system and quality of manufacturing process are the critical roles to ensure and improve the product quality and reliability. Inman et al. [7] believed that performance of manufacturing system severely restricted product quality and reliability, and upgrading the manufacturing equipment or adjusting the technological process could promote the ability of manufacturing system as well as ensuring and optimizing product quality and reliability. To some extent, the ability of trouble free operation of manufacturing system determines the level of inherent reliability formed in manufacturing process.

Traditional reliability modeling of manufacturing system tends to follow the classic reliability block diagram method, fault tree analysis, Petri nets, and so forth, which caused a comprehensive analysis and dynamical assessment for manufacturing system to be complex or inconvenient. Based on the data of system operation and maintenance, $\mathrm{Li}$ and $\mathrm{Ni}$ [8] used the maximum likelihood estimation method to estimate the reliability of manufacturing system, which provided the basis for carrying out preventive maintenance of manufacturing system. Lin and Chang [9] proposed the limited manufacturing network model, and after mining operating failures and rework data, an analysis model of manufacturing system reliability was established. Li et al. [10] created a prediction model of manufacturing system using the grey model, and the author stated that the weaknesses of manufacturing system could be identified by the proposed model. Considering the plenty of quality data existing in manufacturing process, Chen and Jin [11, 12] put forward a Quality-Reliability chain model based on the interaction between manufacturing process quality and manufacturing system reliability, and the reliability analysis and maintenance optimization of manufacturing system were expounded based on the proposed Quality-Reliability chain. Zhang et al. [13] presented a reliability modeling approach of manufacturing system using dimensions of process quality. Rafiee et al. [14] analyzed four typical vibration modes and their effects on the degradation rate of manufacturing process and modeled the complex manufacturing system reliability like MEMS and so on. Regarding the maintenance strategy of manufacturing systems, Li et al. [15] investigated the economic production quantity model jointly considering product deterioration and proposed an EPQ (economical production quantity) model for deteriorating production system and items with rework.
Gong et al. [16] explored an adaptive maintenance model of the process environment to diagnose the progressive faults in manufacturing systems. Tlili et al. [17] proposed a new modeling approach based on the fact that the degradation process is modeled by the wiener process. Hajej et al. [18-21] studied integrated maintenance strategies and policies jointly considering the optimization problems of subcontracting, product returns, lease contract, and so forth, which provide a solid foundation to develop the integrated maintenance strategies optimization for manufacturing system. Mifdal et al. [22] presented a joint optimization approach of maintenance and production planning for a multiple-product manufacturing system, which could establish sequentially an economical production plan and an optimal maintenance strategy considering the influence of the production rate on the system's degradation.

As can be seen from the above literature analysis, the interaction between product inherent reliability and manufacturing system reliability is not defined, and studies on the product reliability oriented reliability modeling and optimization of manufacturing system are few, which are in urgent need to develop reliability assurance in manufacturing. Therefore, in order to promote the joint reliability optimization of manufacturing system and the produced product, an integrated model named RQR chain is proposed by extending the Quality-Reliability chain in this paper, the RQR chain could describe the coeffects of the manufacturing system reliability $R_{m}$, manufacturing process quality $Q_{p}$, and product inherent reliability $R_{p}$, and the impact of manufacturing system reliability on the product inherent reliability is expounded specifically based on product qualified probability. At the same time, the quantitative analysis model and optimization strategies of manufacturing system reliability are given. Comparing to previous related studies in the frame of integrated reliability and maintenance optimization of manufacturing system, the main contributions of this paper are as follows:

(a) RQR chain is brought forth and the product qualified probability is proposed to quantify the impacts of manufacturing process quality on the manufacturing system reliability for the first time. The product qualified probability driven RQR chain could make integrated reliability optimization of manufacturing system and produced product possible.

(b) A reliability modeling approach of manufacturing system based on the proposed qualified probability is presented. The impact of qualified probability on the inherent reliability of produced product oriented is expounded at the first time in this paper, and the concept of the reliability of manufacturing is extended by including the requirement of product quality that is qualified by time $t$ in this proposed approach.

(c) The preventive maintenance optimization strategy for manufacturing system driven by the loss of manufacturing quality variation is proposed by the aid of the product qualified probability. The proposed product qualified probability based optimization approach 
could make the real time preventive maintenance possible when the abnormal quality variations occurred in the produced work pieces in the batch production.

The rest of the paper is organized as follows. Section 2 emphasizes the role of manufacturing process quality variations as a transfer bridge for analyzing the influence of manufacturing system reliability on product inherent reliability, and the RQR chain based on product qualified probability is put forward. Modeling of manufacturing system reliability based on the qualified probability is analyzed in Section 3. With reference to the results of the proposed model, Section 4 presents some optimization strategies driven by product quality loss. Section 5 discusses the application mode and the effects of the proposed method in an automotive cylinder head manufacturing system. Finally, conclusions are drawn in Section 6.

\section{Quality Oriented RQR Chain}

2.1. RQR Chain. In the manufacturing process, normal and abnormal variations from man, machine, material, method, measurement, and environment (5M1E) should be accumulated and inherited by the work pieces, resulting in the variations of product dimensions eventually. Variations of product quality formed in the manufacturing process are the basic factors influencing the product inherent reliability. As manufacturing system acts as the material carrier of manufacturing process, its stability determines the quality of the manufactured product. Thus, it can be concluded to a great extent that the product inherent reliability is relying on the reliability of manufacturing system with a fixed product design scheme, product reliability in manufacturing process would be deteriorated by those abnormal factors like wear degradation or failures of system components or some assembly errors, and so forth, and the number of potential quality defects lying in the manufactured products should increase successively, resulting in the product inherent reliability failing to satisfy the design requirements. It is obvious that if the reliability of manufacturing system cannot be guaranteed effectively, the dimensional parameters of the manufactured work pieces will be deteriorated constantly (such as abnormal dimensional variations in the manufacturing process), and contrasted with design reliability, these variations would bring about a decline of product inherent reliability. To make matters worse, products composed by these unreliable components are prone to some unpredictable fatal failures. In order to describe the relationship among manufacturing system reliability $R_{m}$, process quality $Q_{p}$, and product inherent reliability $R_{p}$, a conceptual model of RQR chain is put forward and shown in Figure 1.

As shown in Figure 1, the product quality in manufacturing process is the linkage of the manufacturing system reliability and product inherent reliability which is clearly reflected by the RQR chain, and in the view of the mathematical modeling, the RQR chain could be divided into the following three layers from the top-down: physical layer, parameter layer, and data layer. The three-tier construction of RQR chain could enable the integrated analysis for the reliability of manufacturing system based on the quality data in manufacturing and reliability data in field as much as possible.

(1) Physical Layer of $R Q R$ Chain. The coeffects of manufacturing system, manufacturing process, and product are described clearly in this layer. Specifically, due to system components wear or failures caused by friction wear, reliability of manufacturing system $R_{m}$ gets lower. During the execution of functional requirements from manufacturing system, quality in manufacturing process $Q_{p}$ results in abnormal variations accompanied by degradation of the manufacturing system. Correspondingly, owing to these quality variations, there may be potential defects retained in the final product, which has a negative effect on product reliability $R_{p}$. That is to say, reliability of manufacturing system determines directly the stability of manufacturing process quality, and then the reliability of produced product is subjected to the stability of manufacturing process quality.

(2) Parameter Layer of $R Q R$ Chain. The indicating parameters of the mentioned coeffects in RQR chain are given in this layer, the quantitative indicators of manufacturing system reliability, product qualified probability, and product inherent reliability are presented, and these parameters are the carriers of the coeffects in RQR chain.

(3) Data Layer of $R Q R$ Chain. The product key quality characteristics including key control characteristics and key product characteristics (KQCs) are presented in the data layer, which provides the data source to compute the value of parameters given in the parameter layer.

As shown in Figure 1, the quality of manufacturing process $Q_{p}$ is the core of the RQR chain, which links manufacturing system reliability $R_{m}$ and product reliability $R_{p}$. To accurately analyze manufacturing system reliability oriented by product inherent reliability and further optimize the manufacturing system, quantifying the quality of manufacturing process is bound to the prerequisite. Typically, current studies adopt product qualified rate to characterize the quality of manufacturing process, which simply reflects the cursory state of process quality $Q_{p}$ in the form of scalar quantity. And it is still actually inconvenient to carry out system reliability $R_{m}$ modeling and optimization that considers process quality variation information in the form of vector. Therefore, based on the definition of product quality, the big data like variation vector of manufacturing process and system components degradation is combined firstly to put forward product qualified probability to represent manufacturing process quality in vector.

2.2. Product Qualified Probability. Product qualified rate is often used in traditional quality control to describe the quality of manufactured products and the capability of manufacturing process. It is deemed that if all the key quality characteristics of products are within their tolerance limits at time $t$, products are thought to pass through the inspection and there is no substandard product throughout the manufacturing process. Count the number of qualified 


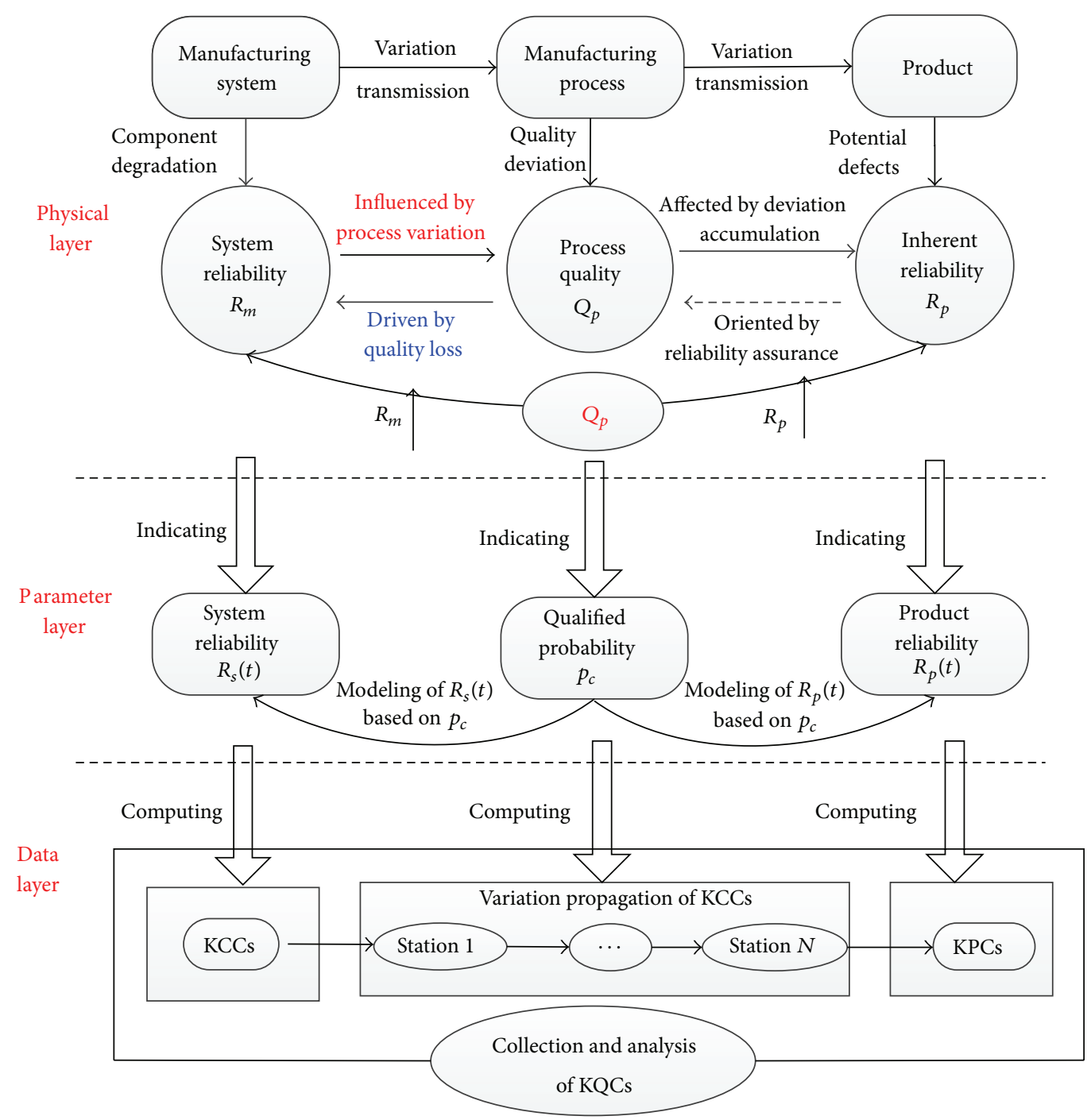

FIgURe 1: Conceptual model of RQR chain.

products and figure out how much it accounts for the total inspected products, and, namely, the product qualified rate is obtained. Here, product qualified rate integrates count information of a single product as a whole and characterizes the quality of manufactured products in the form of scalar. However, the values of the detected quality characteristics are obtained in the vector form with the advancement of detection technology in practical engineering applications usually. Different from the scalar expression of product qualified rate, defining quality levels and grades often requires quantifying the extent of how values of product key quality characteristics approximate the predetermined targets further. And thus, measurable information such as parameters of different components inside a single product should be the concern of quality control. At present the qualified rate is basically the only evidence to determine whether a batch product is qualified or not, and the judgment is arbitrary, which will result in neglecting and omitting useful variation information of those measurable key quality characteristics under modern vectorial measurement environment. And it turns out to be not conducive to fully exploit and utilize process quality data and then carry out a comprehensive analysis of both product reliability and manufacturing system reliability. Accordingly, product qualified probability oriented by quality grade is proposed and the vector space of different parameters that constitute product key quality characteristics is built in this paper, and thus vectorization of qualified degree of products is realized. When values of the key quality characteristics are closer to the predetermined ones, both quality grade and the corresponding degree of qualified product become higher. Namely, it corresponds to a higher product qualified probability. From the perspective of population and sample, Figure 2 contrasts the differences and relations between traditional qualified rate and the proposed qualified probability based on the interactive scalar of the whole population and the vector of the single sample. 


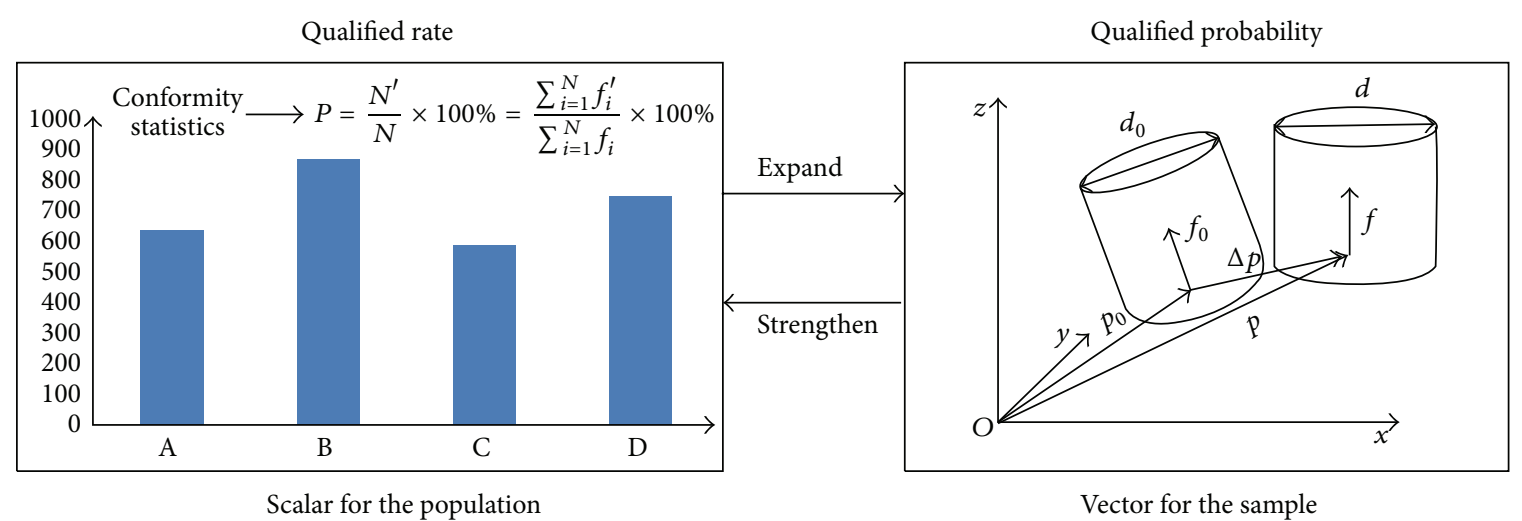

FIGURE 2: Comparison of qualified rate and qualified probability.

Given the threshold value $\theta_{k}$ of key quality characteristics, product qualified probability can be expressed as

$$
P_{k}^{c}=\operatorname{Pr}\left\{\Delta y(k) \leq \theta_{k}\right\} \text {. }
$$

Here, $\Delta y(k)$ denotes the variation of the actual characteristic dimension to the target value at station $k$, and it can be obtained through the SoV [23] (Stream of Variation) theory.

Generally, the threshold value $\theta_{k}$ can be acquired by means of product quality specifications and the popular process capability index. Set the tolerance range of products to be USL (upper specification limit) and LSL (lower specification limit), with process capability index being $C_{\mathrm{pk}}$ (actual process capability) and variance of key quality characteristics being $\theta_{k}$, and their values should be calculated via the formula of $C_{\mathrm{pk}}=(\mathrm{USL}-\mathrm{LSL}) / 6 \sqrt{\theta_{k}}, \theta_{k}=\left[6 C_{\mathrm{pk}} /(\mathrm{USL}-\mathrm{LSL})\right]^{2}$.

Set $f(x)=\Delta y(k)-\theta_{k}$, and ignore the noise of manufacturing process and the measurement. Since $f(x)$ is a nonlinear function of $x(k)=\left[x_{1}, x_{2}, \ldots, x_{n}\right]^{T}$, for simplicity, Taylor expansion is used to linearize $f(x)$ as below:

$$
\begin{aligned}
f(x) & =a_{0}+\sum_{i=1}^{n} a_{i} x_{i} \quad\left(a_{i} \text { is constant }\right), \\
\beta & =\frac{\mu_{f}}{\sigma_{f}} \\
& =-\frac{a_{0}+\sum_{i=1}^{n} a_{i} \mu_{x_{i}}}{\sqrt{\sum_{i=1}^{n} a_{i}^{2} \sigma_{i}^{2}+\sum_{i=1}^{n} \sum_{\substack{j=1 \\
j \neq i}}^{n} a_{i} a_{i} \operatorname{Cov}\left(x_{i}, x_{j}\right)}} \\
& \left(\mu_{f}=a_{0}+\sum_{i=1}^{n} a_{i} \mu_{x_{i}}\right) .
\end{aligned}
$$

As $\mu_{x}=\left(\mu_{x_{1}}, \mu_{x_{2}}, \ldots, \mu_{x_{n}}\right)$ is the mean point of independent variables of $f(x)$, correspondingly, mean of the dependent variable is denoted by $\mu_{f}=f\left(\mu_{x_{1}}, \mu_{x_{2}}, \ldots, \mu_{x_{n}}\right)$ and the standard variation by $\sigma_{f}^{2}=\sum_{i=1}^{n} a_{i}^{2} \sigma_{i}^{2}+$ $\sum_{i=1}^{n} \sum_{\substack{j=1 \\ j \neq i}}^{n} a_{i} a_{i} \operatorname{Cov}\left(x_{i}, x_{j}\right)$. When process variables obey iid (independent and identically distributed), there exists $\sigma_{f}^{2}=\sum_{i=1}^{n} a_{i}^{2} x_{i}^{2}$. And product qualified probability of manufacturing process should be derived as

$$
\begin{aligned}
P_{k}^{c} & =\operatorname{Pr}\left\{\Delta y(k) \leq \theta_{k}\right\}=\operatorname{Pr}\{f(x) \leq 0\} \\
& =p\left\{\frac{f(x)-\mu_{f}}{\sigma_{f}}<-\frac{\mu_{f}}{\sigma_{f}}\right\}=\Phi(-\beta) .
\end{aligned}
$$

\section{Reliability Modeling of Manufacturing System Based on RQR Chain}

3.1. Analysis of Product Inherent Reliability Oriented by Qualified Probability. Manufacturing processes are comprised of raw materials purchasing, parts machining, components assembling, and performance testing. With reference to reliability requirements of the design phase, parts that are either outsourced or homemade are operated by machining, assembling, testing, and so forth, and thus the Work in Process (WIP) or the end products are finally produced. Influenced by a variety of variation factors, the product inherent reliability gets lower than requirements of design reliability usually. Jiang and Murthy [4] noted that nonconforming components and assembly errors would have undesirable effect on the manufactured product reliability, and the nonconforming components are the basic adverse factors. Based on this standpoint, the product qualified probability is brought forward to measure the potential influence of nonconforming components on the product inherent reliability.

Assuming that failure distribution of products in the design phase is $F_{0}(t)$, the design reliability of products $R_{o}(t)$ can be expressed as $R_{o}(t)=1-F_{0}(t)$. Correspondingly, failure density function and failure rate function are denoted by $f_{0}(t)=d F_{0}(t) / d t$ and $r_{0}(t)=f_{0}(t) / R_{o}(t)$, respectively. Similarly in the manufacturing process, failure distribution of nonconforming components is $H(t)$ with reliability function being $R_{h}(t)=1-H(t)$, failure density function being $h(t)=$ $d H(t) / d t$, and failure rate function being $r_{h}(t)=h(t) / R_{h}(t)$. According to the number of the nonconforming components and the unqualified degree of the nonconforming components, define the probability that results in nonconforming components as $q$ and the qualified probability of components as $p_{c}$. 


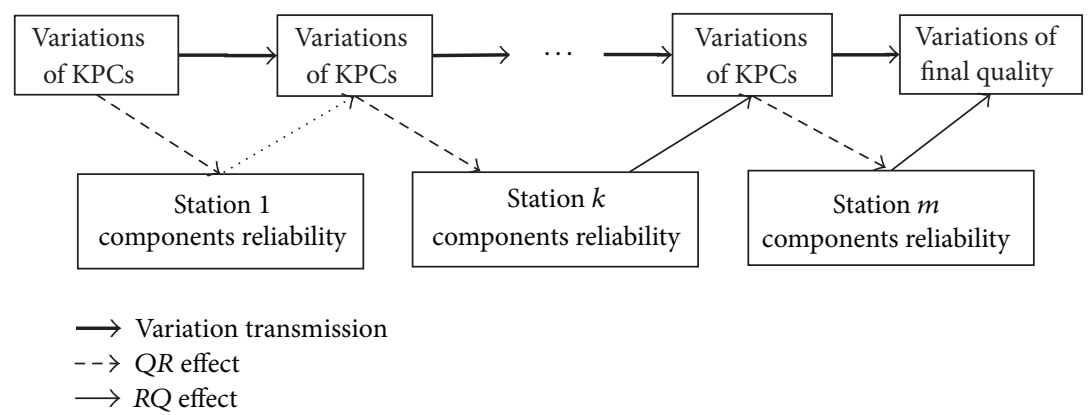

FIGURE 3: Interaction between quality variations and components reliability in multistation process.

Then with all the components being fully qualified, namely, $p_{c}=1$, it is the only case of occurrence of nonconforming components that affects the reliability of manufactured products, which is denoted by

$$
R_{1}(t)=(1-q) R_{o}(t)+q R_{h}(t) .
$$

Obviously, with $q$ being equivalent to 0 , the manufactured reliability equals the design reliability, indicating that no nonconforming components occur, and with $q$ being equivalent to 1 , it happens to be the opposite from the former case of $q=0$, indicating that all components fail to be conforming in manufacturing process.

When it comes to components being not fully qualified with $0<p_{c}<1$, the manufactured reliability is subjected to the occurrence of nonconforming components and the related unqualified level jointly, and, then, inherent reliability of the manufactured product is as follows:

$$
R_{2}(t)=(1-q)\left(1-p_{c}\right) R_{o}(t)+q p_{c} R_{h}(t) .
$$

It is evident that with $p_{c}=1, R_{2}(t)=q R_{h}(t) \leq$ $R_{1}(t)=(1-q) R_{o}(t)+q R_{h}(t)$, which shows that integration of both the occurrence of nonconforming components and their unqualified level is conducive to a more precise estimation of the manufactured reliability. While simply based on the case whether components are unqualified, inherent reliability of the manufactured products will be blindly overestimated, which would have a great negative impact on the objective analysis and optimization for the reliability for manufacturing system.

3.2. Modeling Reliability of Manufacturing System Based on Qualified Probability. Reliability of the manufacturing system is an important factor to ensure product quality and productivity. Considering the difficulty in quantifying the reliability issues timely caused by product dimension variations, usually those reliability issues are easily neglected in system design and optimization. And unfortunately, with many failures or performance degradation failing to be diagnosed in real time, further identification or predication is of nonsense, which may result in unnecessary downtime for machines and reduce the production efficiency. Mechanisms of performance degradation and occurrence of failures of system components are versatile and complicated. Both components themselves of this current station and quality variations from the upstream station make a difference to the performance of components as well as the manufacturing system. Reliability analysis of multistation manufacturing system notes that via the transmission of quality variations, interaction between variations of key product characteristics (KPCs) and reliability of system components is also transferred through the stations. Thus, the statistical correlation between manufacturing system component failures and unqualified products could be shown in Figure 3.

Accordingly, we are badly in need for a new connotation of reliability of manufacturing system. Degree of reliability is generally used to measure the reliability of the manufacturing system which refers to the probability a system completes its intended functions under specified conditions within the specified time. Not only the expected functions of a manufacturing process should consider the uptime of manufacturing system, but also the quality of manufactured product should also be involved to holistically assess the performance and reliability of manufacturing system. As a conclusion, the reliability of manufacturing system $R(t)$ should be identified as the probability that system components do not fail themselves and at the same time manufactured products are completely qualified within a period of time, which can be expressed as

$$
\begin{aligned}
& R(t)=P\{\text { system does not fail by time } t \\
& \quad \cap \text { product quality is qualified by time } t\} .
\end{aligned}
$$

Define $R^{F}$ and $R^{Q}$ to represent cases where system does not suffer catastrophic failures and product quality is qualified by time $t$, respectively. And $Z\left(t_{k}\right)$ means the performance state of system components at the endpoint of $t_{k}$. To sum up, the reliability of manufacturing system could be rewritten as

$R(t)=P\{$ System does not fail by time $t$

$\cap$ Product quality is qualified by time $t\}=\operatorname{Pr}\left\{R^{F}\right.$

$$
\left.\cap R^{Q}\right\}=\operatorname{Pr}\left\{R^{F} \mid Z(t)\right\} \cdot \operatorname{Pr}\left\{R^{Q} \mid Z(t)\right\} .
$$

Here, $\operatorname{Pr}\left\{R^{Q} \mid Z(t)\right\}$ corresponds to the product qualified probability mentioned in Section 2.2. Namely, there exists $\operatorname{Pr}\left\{R^{Q} \mid Z(t)\right\}=P_{k}^{c}=\Phi(-\beta)$. So, the process of how 
to determine the $\operatorname{Pr}\left\{R^{F} \mid Z(t)\right\}$ will be highlighted in the following.

In general, the performance state and relative operating conditions of system components determine the reliability of manufacturing system. Either components failures or degradation by wear could cause a decline in reliability of manufacturing system. Since status information like wear or degradation of tools comes along the running of manufacturing system, failure data may not occur necessarily with the advancement of manufacturing technologies. Therefore, this paper prefers to consider information of wear or degradation of system components as the main factors affecting the reliability of manufacturing system. The wear and tear of system components are accumulated and increased along with the front and back work stations one by one. Let $\Delta(k)$ be the amount of wear from the individual station $k$, and the cumulative amount of wear by station $k$ is $Z(k)=$ $Z(k-1)+\Delta(k)$. If all the wear or degradation processes are independent and identically distributed as most mechanical products, according to the central limit theorem, $Z(k)$ can be rewritten as

$$
Z(k)=\sum_{j=1}^{k} \Delta(j) \approx N(k \cdot E[\Delta(j)], k \cdot \operatorname{Var}[\Delta(j)]) .
$$

Define $\lambda_{k}(t)$ as the probability that system component fails at station $k+1$ while it still functions well at station $k$ by time $t$, namely, the failure rate of the system component. Assuming that failure of the individual component is subjected to an exponential distribution (for high reliability of complex systems, exponential distribution can approximately model the failure distribution for those system components), reliability of system components at station $k$ is expressed as (excluding the impact of component wear on component strength, the failure rate can be regarded as a constant):

$$
R_{k}^{F}(t)=e^{-\lambda_{k}(t) \cdot t}
$$

When the input products have problems of quality variations, wear and tear of system components will be accelerated as

$$
\lambda_{k}(t)=\lambda_{0}(t)+E\left(\alpha_{k}\left(X(k)-m_{k}\right)^{2}\right) .
$$

Here, $\lambda_{0}(t)$ is the initial failure rate irrespective of impact of input product quality on system components; $X(k)$ is the practical quality index at station $k ; m_{k}$ stands for the standard value of $X(k)$ with $\alpha_{k}$ being the correction coefficient which reflects the impact of input quality variation on wear of components. And the reliability of the whole system could be presented as

$$
R^{F}(t)=\prod_{i=1}^{n} R_{i}^{F}(t)
$$

Suppose that $\eta$ is the allowable maximum amount of components wear, $\varepsilon_{k}$ is the degradation coefficient of relevant performance, $w_{0}$ is the initial rate of degradation which corresponds to time $t_{k}=0$, and the probability that no failures occurred for the whole system by time $t$ is as follows:

$$
\begin{aligned}
\operatorname{Pr} & \left\{R^{F} \mid Z(t)\right\}=P\left(\prod_{i=1}^{n} R_{i}^{F} \mid Z(t)<\eta\right) \\
& =P\left(\prod_{i=1}^{n} e^{-\left[\lambda_{0 i}(t)+E\left(\alpha_{i}\left(X(i)-m_{i}\right)^{2}\right)\right] \cdot t_{i}} \mid \sum_{k=0}^{h} w(t)<\eta\right) \\
& =P\left(\prod_{i=1}^{n} e^{-\left[\lambda_{0 i}(t)+E\left(\alpha_{i}\left(X(i)-m_{i}\right)^{2}\right)\right] \cdot t_{i}} \mid h\left(w_{0}+e^{-\varepsilon_{k} \cdot t}\right)\right. \\
& <\eta \\
& =\prod_{i=1}^{n} \exp \left(-\left[\lambda_{0 i}(t)+E\left[\alpha_{i}\left(X(i)-m_{i}\right)^{2}\right]\right] \cdot t_{i}\right) \\
& \cdot \int_{0}^{\eta} \exp \left(-h\left(w_{0}+e^{-\varepsilon_{k} \cdot t}\right)\right) d \varepsilon .
\end{aligned}
$$

Combined with formula (8), the final expression of the reliability of manufacturing system based on the product qualified probability $R(t)$ could be written as

$$
\begin{aligned}
R(t) & =\operatorname{Pr}\left\{R^{F} \mid Z(t)\right\} \times \operatorname{Pr}\left\{R^{Q} \mid Z(t)\right\} \\
& =\exp \left(\sum_{i=1}^{n}\left(-\left[\lambda_{0}(t)+E\left[\alpha_{i}\left(X(i)-m_{i}\right)^{2}\right]\right] \cdot t\right)\right) \\
& \cdot \int_{0}^{\eta} \exp \left(-h\left(w_{0}+e^{-\varepsilon_{k} \cdot t}\right)\right) d \varepsilon_{k} \\
\cdot & \Phi\left(-\frac{\alpha_{0}+\sum_{i=1}^{n} \alpha_{i} \mu_{x_{i}}}{\sqrt{\sum_{i=1}^{n} \alpha_{i}^{2}+\sum_{i=1}^{n} \sum_{\substack{j=1 \\
j \neq i}}^{n} \alpha_{i} \alpha_{i} \operatorname{Cov}\left(x_{i}, x_{j}\right)}}\right) \\
& =\exp \left(\sum_{i=1}^{n}\left(-\left[\lambda_{0}(t)+E\left[\alpha_{i}\left(X(i)-m_{i}\right)^{2}\right]\right] \cdot t\right)\right) \\
& \cdot \int_{0}^{\eta} \exp \left(-h\left(w_{0}+e^{-\varepsilon_{k} \cdot t}\right)\right) d \varepsilon_{k} \Phi(-\beta) .
\end{aligned}
$$

According to formula (14), if the interaction between product quality and components failures or performance degradation has been ignored, the reliability of manufacturing system should be overestimated, which would endanger the quality and reliability of manufactured products, and the final goal to obtain high reliable products cannot be fulfilled. The correlation modeling of product quality and components reliability should help us to establish a more objective and accurate model, which makes a more authentic and practical assessment of the reliability of manufacturing system, and provides specific goals and directions for further improving the reliability of manufacturing system. 


\section{Preventive Optimization Strategy for Reliability of Manufacturing System Driven by Quality Loss in Manufacturing Process}

4.1. Requirements Analysis of Dynamic Optimization. The purposes of conducting analysis, modeling, and assessment of the reliability manufacturing system are mainly designed to guide the timely maintenance for failed components or degraded ones, which would help to improve the quality level of the manufactured products, and how to obtain the optimal strategy in real time of the reliability of manufacturing system has long been a mathematical puzzle in industrial engineering $[16,17]$.

The previously proposed reliability model of manufacturing system highlights the role of product quality variation and its interaction effect with components reliability. Therefore, not only maintenance cost of manufacturing system itself but also the quality loss in manufacturing process should be simultaneously considered when developing reliability optimizing strategies for manufacturing system. That is to say, the total costs for optimizing manufacturing system should include quality loss caused by the variations that occurred in manufacturing process, maintenance costs by component failures, and so forth. According to the reliability model presented in Section 3.2, in order to realize dynamic or real time optimization, the product quality loss should be taken into account to formulate the optimization strategies. For the convenience of the computation, the assumptions and hypotheses are given firstly as below:

(1) Suppose the maintenance cycle is $T$, and $T=$ $k \Delta t, k \in N$, where $k$ is a constant. Then the extent of performance degradation for each component can be determined. Denote the degradation state of component $i$ by $z_{i}(i=1,2, \ldots, n)$ and assume that the cost needed for conducting a state inspection for component $i$ is $C_{i}$, where the inspection time can be neglected. Without loss of generality, $\Delta t$ can be considered as 1 , and thus $T$ can be directly written as $k$ in the context. Since the operating time for each component may be different from each other, time for state inspection separately may be not necessarily synchronized.

(2) Assume there are two ways of maintenance of each component, which includes the postmaintenance and preventive maintenance, and inspection time of the maintenance is fixed at $t_{k}$. If the maintenance is assumed to be an overhaul or a complete replacement, functions of components are believed to be fully restored by repairment. Define costs for postmaintenance and preventive maintenance as $C_{c}^{i}$ and $C_{p}^{i}$, respectively, where the magnitude relation conforms to $C_{c}^{i}>C_{p}^{i}$.

(3) To quantify the impact of components degradation on product quality in manufacturing process, Taguchi function is adopted to analyze the quality loss caused by components degradation or error propagation, and so forth.
4.2. Optimization Strategy Decision-Making Model Driven by Quality Loss. Based on the above analysis and assumptions, the objective of optimization strategy for the reliability of manufacturing system is to minimize the costs of quality loss, postmaintenance, and preventive maintenance simultaneously. And it can be represented as a constraint optimization problem in the following expression:

$$
\operatorname{Min} \mathrm{EC}=\operatorname{Min} f\left(C_{m}, C_{q}\right)
$$

where EC represents the expected cost of the optimization strategy, $C_{m}$ means the expectation of average maintenance costs comprised of postmaintenance costs and preventive maintenance costs, and $C_{q}$ signifies the expectation of average quality loss.

It is generally believed that failure rate of each system component is fixed to the same as $\lambda_{i}$. And to be consistent with the prementioned assumption in Section 3.2, the failure of the individual component is subjected to an exponential distribution, and the reliability of system components at station $i$ is expressed as $e^{-\lambda_{i} \cdot t}$. Accordingly, the preventive maintenance at the unit expense of $C_{p}^{i}$ for the reliable system component gets the cost of $C_{p}^{i} e^{-\lambda_{i} t}$, whereas the postcorrective maintenance at the unit expense of $C_{c}^{i}$ for the unreliable system component gets the cost of $C_{c}^{i}(1-$ $\left.e^{-\lambda_{i} t}\right)$. And thus, for one single system component by each inspection time, the total maintenance cost generally consists of the two mentioned parts of the preventive $C_{p}^{i} e^{-\lambda_{i} t}$ and the postcorrective $C_{c}^{i}\left(1-e^{-\lambda_{i} t}\right)$.

What is more, with lifetime of component $i$ being $s_{i}$ and the number of these system components being $n$, the average maintenance costs $C_{m}$ can be extended as

$$
C_{m}=\sum_{i=1}^{n} \frac{C_{p}^{i} e^{-\lambda_{i} t}+C_{c}^{i}\left(1-e^{-\lambda_{i} t}\right)}{\int_{0}^{s_{i}} e^{-\lambda_{i} t} d t} .
$$

And it can be simplified as the following expression:

$$
C_{m}=\sum_{i=1}^{n} \lambda_{i}\left[\frac{C_{p}^{i}}{1-e^{-\lambda_{i} s_{i}}}+C_{p}^{i}-C_{c}^{i}\right]
$$

And the quality loss function based on the Taguchi function is expressed as

$$
L(k)=q\left(X(k)-m_{k}\right)^{2} .
$$

Here, $L(k)$ is the quality loss with $q$ the constant responding to the coefficient of quality loss, $X(k)$ representing product key quality characteristic, and $m_{k}$ being the specification value for $X(k)$.

Take the mathematical expectation for formula (18) as

$$
E[L(k)]=q \operatorname{Var}[X(k)]+q\left[E(X(k))-m_{k}\right]^{2},
$$

wherein $E[X(k)]$ and $\operatorname{Var}[X(k)]$ are the mathematical expectation and variance of $X(k)$, respectively. 


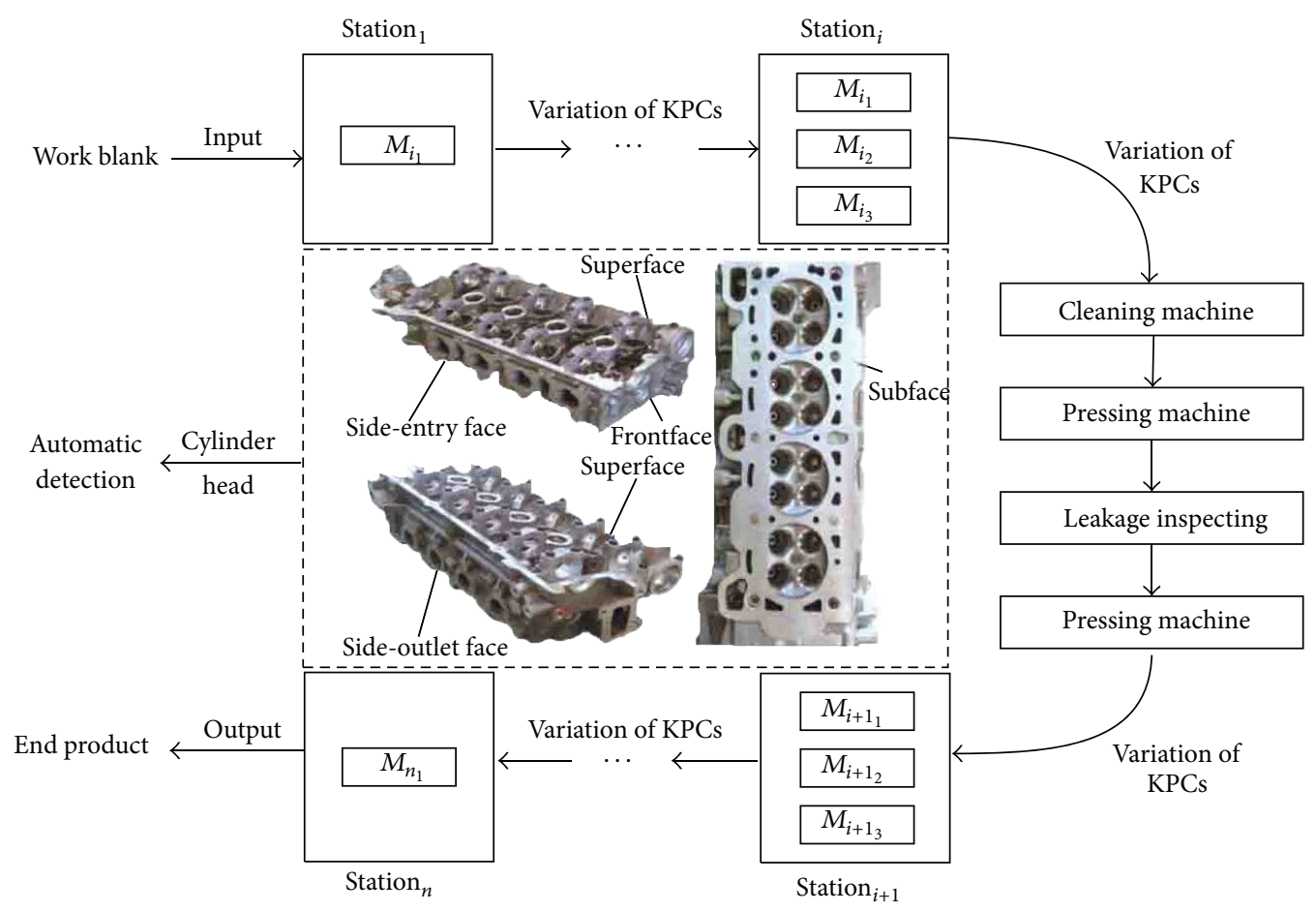

FIGURE 4: An illustration of key manufacturing process for cylinder head manufacturing system.

When quality loss is taken into account of the optimization costs, estimation for the costs will be more conservative actually. After applying the exponential distribution to describe cost of quality loss by time $t$, the expectation of average quality loss $C_{q}$ should be rewritten as

$$
C_{q}(t)=C_{0} \exp \{-E[L(k)] \cdot t\},
$$

where $C_{0}$ is the initial cost, meaning there is no loss of product quality.

Integrating formula (16) and formula (20) into formula (15), the optimization objective is converted to minimize both maintenance costs and product quality loss, and the final formula is as follows:

$$
\begin{gathered}
\operatorname{Min} \mathrm{EC}=\operatorname{Min} f\left(\sum_{i=1}^{n} \lambda_{i}\left[\frac{C_{p}^{i}}{1-e^{-\lambda_{i} s_{i}}}+C_{p}^{i}-C_{m}^{i}\right],\right. \\
\left.\lim _{T \rightarrow \infty} \frac{\int_{0}^{T} C_{0} \exp \{-E[L(k)] \cdot t\} d t}{T}\right) .
\end{gathered}
$$

\section{Case Study}

5.1. Background. As a key part of the engine, the cylinder head is mounted on the upper end of the cylinder block with cylinder head bolts, forming a sealed combustion chamber together with the cylinder block. Coordinating with components of intake and exhaust valves, fuel injectors, pneumatic valves and others, the cylinder head plays a vital role in controlling fully combustion of the air and fuel inside the cylinder. And thus, the system and process for manufacturing the cylinder head turns to be particularly complex and elaborated, key quality characteristics like the surface roughness, geometrical shape, machining dimensions and location precision are needed special attention and monitoring. Usually, variations of dimensions have a great impact on the assembly precision and even the overall performance of the engine. The cylinder head is comprised of the following six components: a superface, a subface, a frontface, a backface, a side-entry face, and a side-outlet face. The machining features are reflected in the complicated structures of surfaces and holes. Accordingly, how to assure the machined surfaces and holes with high precision is the core function of the manufacturing system of cylinder heads, which includes function modules of cutting, clamping, controlling, testing, and clearing. The key operation processes of the cylinder head manufacturing system are shown in Figure 4.

In this paper, key design characteristics of the cylinder head machined by the studied manufacturing system are listed in Table 1.

In practical manufacturing process, due to the integrated effects of various variations, reliability of the cylinder head fails to meet the designed reliability requirements, resulting in a phenomenon of reliability degradation relative to the design reliability and an unsatisfactory response from customers. With mechanical inspection highly automated, it is urgent to reduce the interference from the vector space of key product characteristics and further to monitor the cylinder head quality effectively throughout the entire manufacturing process. Typically, optimization of manufacturing system reliability is the fundamental premise to control the cylinder 
TABLE 1: Key design characteristics of the cylinder head.

\begin{tabular}{lcccc}
\hline Basic dimension & Machining equipment & Process description & Major characteristics & Design specification \\
\hline$B_{1}$ & Machining center & Drilling hole A & Diameter & $\phi 13_{0}^{+0.018}$ \\
$B_{2}$ & Machining center & Drilling hole B & Diameter & $\phi 12.5_{0}^{+0.018}$ \\
$B_{3}$ & Horizontal machine center & Milling face C & Distance & $121.3 \pm 0.1$ \\
$B_{4}$ & Horizontal machine center & Hinging hole D & Diameter & $\phi 10_{0}^{+0.15}$ \\
\hline
\end{tabular}

head quality and reliability. However, whether to replace worn tools or conduct regular maintenance for a high level of system reliability, the level of qualified rate and reliability of the produced products still remained low. How to conduct the reliability of manufactured product oriented modeling and optimizing of manufacturing system reliability are what the proposed RQR chain tries to contribute.

With characteristics of $B_{1}, B_{2}, B_{3}, B_{4}$ identified as the key product characteristics, interaction of process product quality and system components reliability is established and the associated model of manufacturing system reliability and product quality oriented by product qualified probability is then created. Correspondingly, it is proved to play an important role in the reduction of dimension variations of the cylinder head, the decrease of tooling adjustments, the increase of manufacturing system reliability, and the drop in risk of degradation. Specific modeling processes are shown in Figure 5.

5.2. Numerical Example. As shown in Figure 5, reliability analysis and the optimization example for the cylinder head manufacturing systems based on the proposed RQR chain are conducted in the following steps.

Step 1. Create the vector space of key product characteristics as $X_{i}\left(p_{i x}, p_{i y}, p_{i z}, n_{i x}, n_{i y}, n_{i z}\right)$ and ascertain the key feature dimensions of $Y_{k}$. To be specific, in order to adapt the automatic detection process, the position vector $p_{i}$ and the orientation vector $n_{i}(i=1,2,3,4)$ are defined, respectively, to quantify key characteristics of $B_{1}, B_{2}, B_{3}, B_{4}$ facing the three-dimensional space. Table 2 presents the parameters which the vector space $X_{i}\left(p_{i x}, p_{i y}, p_{i z}, n_{i x}, n_{i y}, n_{i z}\right)(i=$ $1,2,3,4)$ contains.

Based on the theory of SoV [23], transmission of key product dimensions between stations in manufacturing system is simplified as shown in Figure 6.

With the major variation $u_{k}$ and noise of production $w_{k}(k=1,2,3,4)$ considered and regardless of noise of measurement $\gamma_{k}$, the produced product dimensions can then be expressed as

$$
\begin{aligned}
& x(k)=A(k) x(k-1)+B(k) u_{k}, \\
& y(k)=C(k) x(k) .
\end{aligned}
$$

Parameters of $A(k), B(k)$, and $C(k)$ in formula (22) are ascertained by variation data of key product dimensions. With reference to what Table 2 has exhibited, use Mathematica software to calculate the four key dimensions as $y(k)=$ $[13.01,12.496,121.18,10.07]^{T}$.
TABLE 2: Vector space model of key product characteristics.

\begin{tabular}{lccccccc}
\hline Number & Key characteristics & $n_{x}$ & $n_{y}$ & $n_{z}$ & $p_{x}$ & $p_{y}$ & $p_{z}$ \\
\hline 1 & Hole A & 0 & 1 & 0 & 41.5 & 22.5 & 0 \\
2 & Hole B & 0 & 1 & 0 & 14.5 .5 & 22.5 & 0 \\
3 & Face C & 0 & -1 & 0 & 91.5 & -15.5 & 0 \\
4 & Hole D & 1 & 0 & 0 & 0 & 0 & 61.5 \\
\hline
\end{tabular}

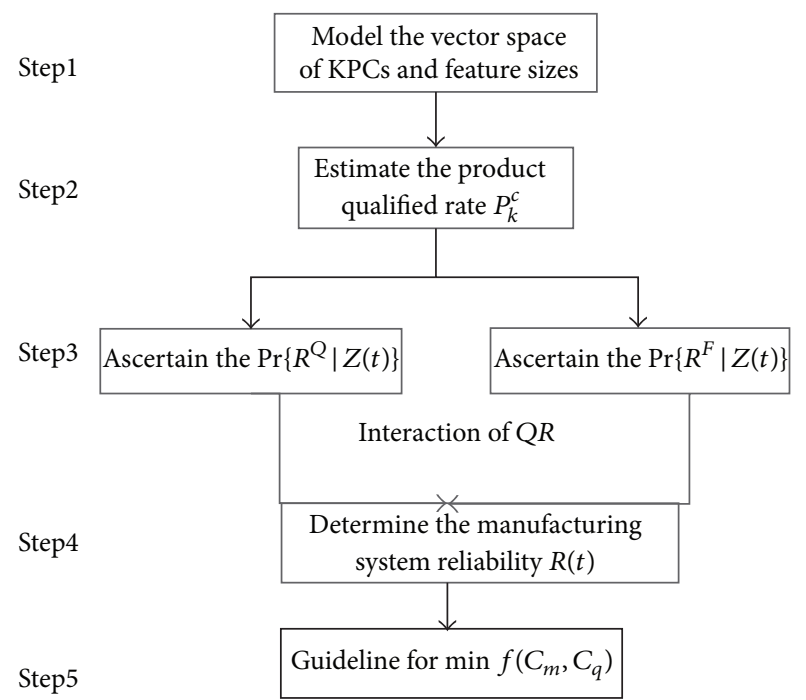

FIGURE 5: Flowchart of modeling and optimization.

Step 2. According to the obtained key dimensions $Y_{k}$, determine the product qualified probability $P_{k}^{c}$ of manufacturing process and the impact $\operatorname{Pr}\left\{R^{Q} \mid Z(t)\right\}$ of process quality $Q$ on reliability of manufacturing system. Assume that the observed original data follow a normal distribution, the related covariance becomes $\sum_{i=1}^{4} \sum_{\substack{j=1 \\ j \neq i}}^{4} a_{i} a_{i} \operatorname{Cov}\left(x_{i}, x_{j}\right)=0$ and $\mu_{f}$ and $\sigma_{f}^{2}$ are as follows:

$$
\begin{aligned}
& \mu_{f}=f\left(\mu_{x_{1}}, \mu_{x_{2}}, \mu_{x_{3}}, \mu_{x_{4}}\right)=0.382, \\
& \sigma_{f}^{2}=\sum_{i=1}^{4} a_{i}^{2} \sigma_{i}^{2}+\sum_{i=1}^{4} \sum_{\substack{j=1 \\
j \neq i}}^{4} a_{i} a_{i} \operatorname{Cov}\left(x_{i}, x_{j}\right)=0.3454 .
\end{aligned}
$$

Referring to formula (3), the value of $\beta$ should be computed as follows:

$$
\beta=\frac{\mu_{f}}{\sigma_{f}}=\frac{0.382}{\sqrt{0.3454}}=0.65 .
$$




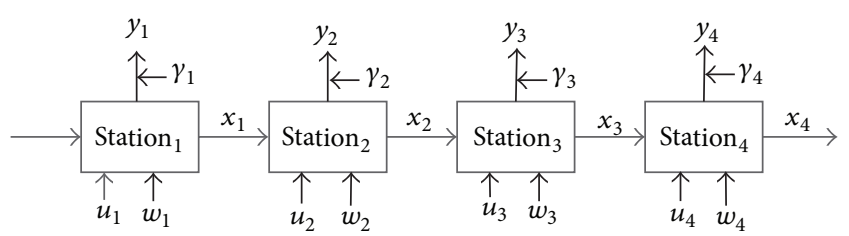

FIGURE 6: Simplified transmission model of key product dimensions.

And thus the product qualified probability is computed as

$$
\operatorname{Pr}\left\{R^{Q} \mid Z(t)\right\}=\Phi(\beta)=\Phi(0.65)=0.7422 .
$$

Step 3. Determine the impact of components reliability on manufacturing system reliability $\operatorname{Pr}\left\{R^{F} \mid Z(t)\right\}$ with process quality $Q$, wear loss, and failure rates of system components quantified, given that the fading rates $w\left(t_{k}\right)=w_{0}+\exp \left(-\varepsilon_{k} \cdot t_{k}\right)$ are parameterized with $w_{0}=5 \times 10^{-5}$ and $\varepsilon_{k}=1 \times 10^{-3}$. Meanwhile, the initial failure rate of the system component is $\lambda_{0 i}=6 \times 10^{-6}(i=1,2,3,4)$ and the interaction coefficients are $\alpha_{k}=3 \times 10^{-4}, h=500$, and $\eta=1.3 \times 10^{-2}$. Set the work time $t$ for each station as 200 hours identically. With reference to formula (13), $\operatorname{Pr}\left\{R^{F} \mid Z(t)\right\}$ can be calculated as

$$
\begin{aligned}
\operatorname{Pr} & \left\{R^{F} \mid Z(t)\right\} \\
& =\exp \left(\sum_{i=1}^{n}\left(\left[\lambda_{0}(t)+E\left[\alpha_{i}\left(X(i)-m_{i}\right)^{2}\right]\right]\right) \cdot t_{i}\right) \\
& \cdot \int_{0}^{\eta} \exp \left(-h\left(w_{0}+e^{-\varepsilon_{k} \cdot t}\right)\right) d \varepsilon_{k}=0.871 .
\end{aligned}
$$

Step 4. Based on the product qualified probability $P_{k}^{c}$ and information of components reliability, the analysis model $R(t)=\operatorname{Pr}\left\{R^{F} \cap R^{Q}\right\}$ for manufacturing system reliability oriented by product inherent reliability is established. Integrate information of process quality $\operatorname{Pr}\left\{R^{Q} \mid Z(t)\right\}$ and components reliability into formula (14), and the reliability of manufacturing system $R(t)$ is finally estimated as

$$
\begin{aligned}
& R(t)=\operatorname{Pr}\left\{R^{F} \mid Z(t)\right\} \times \operatorname{Pr}\left\{R^{Q} \mid Z(t)\right\} \\
& \quad=\exp \left(\sum_{i=1}^{n}\left(-\left[\lambda_{0}(t)+E\left[\alpha_{i}\left(X(i)-m_{i}\right)^{2}\right]\right] \cdot t\right)\right) \\
& \quad \cdot \int_{0}^{\eta} \exp \left(-h\left(w_{0}+e^{-\varepsilon_{k} \cdot t}\right)\right) d \varepsilon_{k} \Phi(-\beta) \\
& =0.871 \times \Phi\left(\frac{0.382}{\sqrt{0.3454}}\right)=0.871 \times 0.7422=0.646 .
\end{aligned}
$$

Step 5. Based on the above results, optimization strategy involved of process quality loss for manufacturing system is analyzed quantitatively. The intended idea is based on the comparison of the difference between the estimated $R_{\mathrm{FQ}}$ from the correlation model, namely, the $\operatorname{Pr}\left\{R^{F} \cap R^{\mathrm{Q}}\right\}$, and the estimated $R_{F}$ irrespective of the correlation between process quality and components reliability, namely, the $\operatorname{Pr}\left\{R^{F} \mid Z(t)\right\}$.

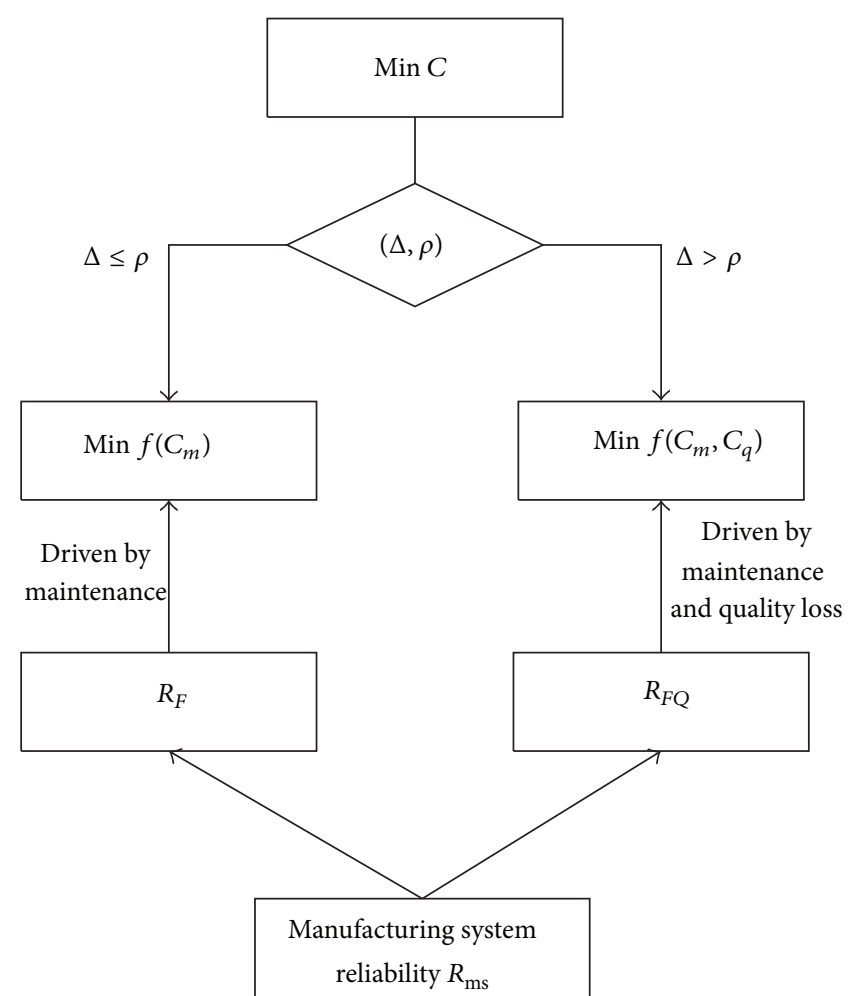

FIGURE 7: Optimization strategy for the manufacturing system.

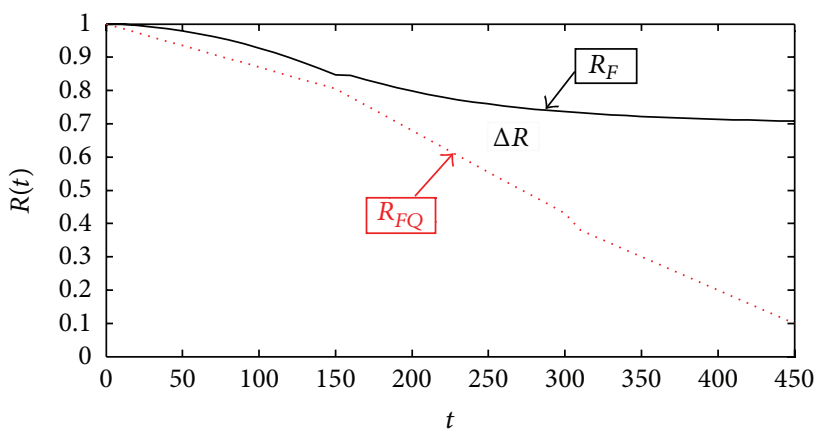

FIGURE 8: Comparison of manufacturing system reliability for the cylinder head.

With the difference $\Delta=R_{F}-R_{F Q}$ and the threshold value $\rho$ ascertained, optimization strategy for manufacturing system is shown in Figure 7.

5.3. Result Analysis. From the results shown in Section 5.2, it is obvious that when the correlation between process quality and components reliability is not considered, the reliability of manufacturing system is approximately 0.871 , whereas it turns to be 0.646 when the correlation is considered. This is to say, manufacturing system reliability is overestimated by $38.9 \%$.

Choose time $t$ as the independent variable; comparison between $R_{F}$ and $R_{F Q}$ is shown in Figure 8 .

As shown in Figure 8, the system reliability is often overestimated when we do not consider the interaction between 


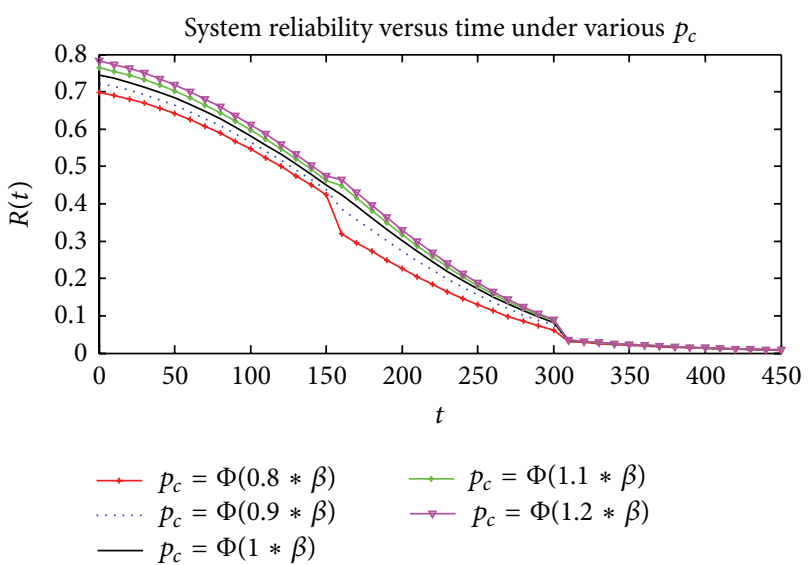

FIGURE 9: Sensitivity analysis of $R$ on $p_{c}$ for the cylinder head.

product quality and components degradation or failures. Through the integral operation, the overestimated reliability is $44.97 \%$. Additionally, the estimation error becomes more significant as time increases, which indicates that the cumulative transmission of quality variations does have a profound effect on the system reliability. Furthermore, in order to show the robustness of the proposed approach, sensitivity analysis of the reliability of manufacturing system $R$ on the product qualified probability $p_{c}$ is simulated, and the result is shown in Figure 9.

As shown in Figure 9, five levels of $p_{c}$ have been set to analyze the significant impact of the cylinder head qualified probability on the reliability of the manufacturing system $R(t)$. With a series of $\pm 20 \%$ shift from the basic $\operatorname{Pr}\left\{R^{Q}\right.$ | $Z(t)\}=\Phi(\beta)=\Phi(0.65)=0.7422$, namely, $p_{c}=$ $\Phi(0.8 * \beta), p_{c}=\Phi(0.9 * \beta), p_{c}=\Phi(1 * \beta), p_{c}=$ $\Phi(1.1 * \beta)$, and $p_{c}=\Phi(1.2 * \beta)$, five reliability curves of the manufacturing system are plotted versus time $t$ under different levels of the product qualified probability $p_{c}$ to demonstrate the correlated sensitivity and robustness. And the synthetical simulation result shows that a high level of the proposed cylinder head qualified probability can usually guarantee a high level of the system reliability. Accordingly, the model of manufacturing system reliability based on the product qualified probability would help the reliability engineer to form a more objective and accurate mathematical model to analyze comprehensively the reliability and performance for manufacturing system. Moreover, the appropriate optimization strategies covering the maintenance for system components and adjustment of process scheme can be got, which should provide clear goals and directions to continuously improve the manufacturing system reliability.

\section{Conclusion}

In this paper, the RQR chain which could fuse the quality data and reliability data in manufacturing system reliability optimization is put forward, and the product qualified probability is introduced to quantify the impacts of quality variation in manufacturing process on the reliability of manufacturing system at the first time. Furthermore, a novel mathematical model of manufacturing system reliability is established based on the product qualified probability and RQR chain. Finally, the reliability and maintenance optimization strategy for manufacturing system is analyzed in view of total cost of maintenance costs and quality loss. The application result demonstrated that manufacturing system reliability tends to be overestimated if the mentioned interaction between product quality and manufacturing system reliability is omitted. The overrated value of the reliability of manufacturing system will not only deteriorate the produced product reliability and may also lead to a wrong maintenance strategy or miss the best opportunity for system maintenance.

To conclude, it is critically essential to consider the coeffects between process quality and system components reliability when modeling and assessing the reliability of manufacturing system. For future research, the following topics should be further expounded.

(1) The improvement of the reliability optimization model based on the quality loss for different type of manufacturing system is needed. The coefficients of the optimization model are different for different manufacturing system; therefore, how to estimate accurately the coefficients from the big data from manufacturing system design, operation, and maintenance is planned.

(2) The quantitative mathematical relationship of the manufacturing system reliability, manufacturing process quality, and the produced product reliability should be established successively. Specifically, the mathematical impact of the manufacturing system reliability on the produced product reliability should be constructed clearly, which should provide a solid foundation for the integrated reliability and maintenance optimization framework of the various types of manufacturing system.

(3) In the last perspective research, we consider the aspect of reliability modeling and assessment in the design and setup of manufacturing system. The reliability level is determined in the design process of the manufacturing system, in order to satisfy the everincreasing stringent quality and reliability requirements, the reliability design should be integrated with the functional design of manufacturing system, and new design theory like Axiomatic Design should be adopted into reliability design of manufacturing system.

\section{Conflict of Interests}

The authors declare that there is no conflict of interests regarding the publication of this paper.

\section{Acknowledgment}

This research was supported by Grant 61473017 from the National Natural Science Foundation of China. 


\section{References}

[1] Y. He, W. Linbo, Z. He, and M. Xie, "A fuzzy TOPSIS and rough set based approach for mechanism analysis of product infant failure," Engineering Applications of Artificial Intelligence, 2015.

[2] M. Colledani, T. Tolio, A. Fischer et al., "Design and management of manufacturing systems for production quality," CIRP Annals: Manufacturing Technology, vol. 63, no. 2, pp. 773-796, 2014.

[3] P. Murthy, "New research in reliability, warranty and maintenance," in Proceedings of the 4th Asia-Pacific International Symposium on Advanced Reliability and Maintenance Modeling (APARM '10), pp. 504-515, McGraw-Hill International Enterprises, Wellington, New Zealand, December 2010.

[4] R. Jiang and D. N. P. Murthy, "Impact of quality variations on product reliability," Reliability Engineering \& System Safety, vol. 94, no. 2, pp. 490-496, 2009.

[5] J. Li and N. Huang, "Quality evaluation in flexible manufacturing systems: a Markovian approach," Mathematical Problems in Engineering, vol. 2007, Article ID 57128, 24 pages, 2007.

[6] J. Li and J. Lei, "Integration of manufacturing system design and quality management," IIE Transactions, vol. 45, no. 6, pp. 555556, 2013.

[7] R. R. Inman, D. E. Blumenfeld, N. Huang, J. Li, and J. Li, "Survey of recent advances on the interface between production system design and quality," IIE Transactions, vol. 45, no. 6, pp. 557-574, 2013.

[8] N. Li and J. Ni, "Reliability estimation based on operational data of manufacturing systems," Quality and Reliability Engineering International, vol. 24, no. 7, pp. 843-854, 2008.

[9] Y.-K. Lin and P.-C. Chang, "System reliability of a manufacturing network with reworking action and different failure rates," International Journal of Production Research, vol. 50, no. 23, pp. 6930-6944, 2012.

[10] G.-D. Li, S. Masuda, D. Yamaguchi, and M. Nagai, "A new reliability prediction model in manufacturing systems," IEEE Transactions on Reliability, vol. 59, no. 1, pp. 170-177, 2010.

[11] Y. Chen and J. Jin, "Quality-reliability chain modeling for system-reliability analysis of complex manufacturing process," IEEE Transactions on Reliability, vol. 54, no. 3, pp. 475-488, 2005.

[12] Y. Chen and J. Jin, "Quality-oriented-maintenance for multiple interactive tooling components in discrete manufacturing processes," IEEE Transactions on Reliability, vol. 55, no. 1, pp. 123134, 2006.

[13] F. Zhang, J. Lu, Y. Yan, S. Tang, and C. Meng, "Dimensional quality oriented reliability modeling for complex manufacturing process," International Journal of Computational Intelligence Systems, vol. 4, no. 6, pp. 1262-1268, 2011.

[14] K. Rafiee, Q. Feng, and D. W. Coit, "Reliability modeling for dependent competing failure processes with changing degradation rate," IIE Transactions, vol. 46, no. 5, pp. 483-496, 2014.

[15] N. Li, F. T. S. Chan, S. H. Chung, and A. H. Tai, "An EPQ model for deteriorating production system and items with rework," Mathematical Problems in Engineering, vol. 2015, Article ID 957970, 10 pages, 2015.

[16] X. Gong, Y. Feng, H. Zheng, and J. Tan, "An adaptive maintenance model oriented to process environment of the manufacturing systems," Mathematical Problems in Engineering, vol. 2014, Article ID 537452, 10 pages, 2014.
[17] L. Tlili, M. Radhoui, and A. Chelbi, "Condition-based maintenance strategy for production systems generating environmental damage," Mathematical Problems in Engineering, vol. 2015, Article ID 494162, 12 pages, 2015.

[18] Z. Hajej, N. Rezg, and A. Gharbi, "Forecasting and maintenance problem under subcontracting constraint with transportation delay," International Journal of Production Research, vol. 52, no. 22, pp. 6695-6716, 2014.

[19] H. Zied, D. Sofiene, and R. Nidhal, "Joint optimisation of maintenance and production policies with subcontracting and product returns," Journal of Intelligent Manufacturing, vol. 25, no. 3, pp. 589-602, 2014.

[20] Z. Hajej, N. Rezg, and A. Gharbi, "A decision optimization model for leased manufacturing equipment with warranty under forecasting production/maintenance problem," Mathematical Problems in Engineering, vol. 2015, Article ID 274530, 14 pages, 2015.

[21] Z. Hajej, S. Turki, and N. Rezg, "Modelling and analysis for sequentially optimising production,maintenance and delivery activities taking into account product returns," International Journal of Production Research, vol. 53, no. 15, pp. 4694-4719, 2015.

[22] L. Mifdal, Z. Hajej, and S. Dellagi, "Joint optimization approach of maintenance and production planning for a multipleproduct manufacturing system," Mathematical Problems in Engineering, vol. 2015, Article ID 769723, 17 pages, 2015.

[23] J. Shi, Stream of Variation Modeling and Analysis for Multistage Manufacturing Processes, CRC Press, Taylor \& Francis, 2007. 


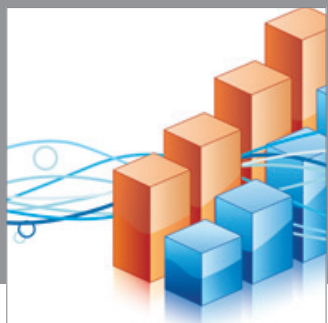

Advances in

Operations Research

mansans

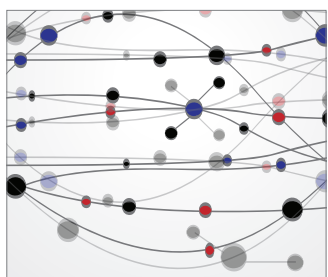

The Scientific World Journal
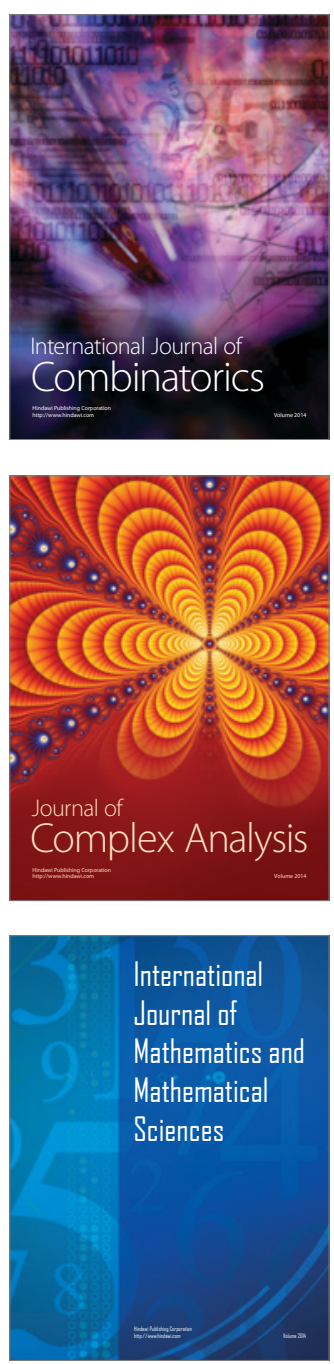
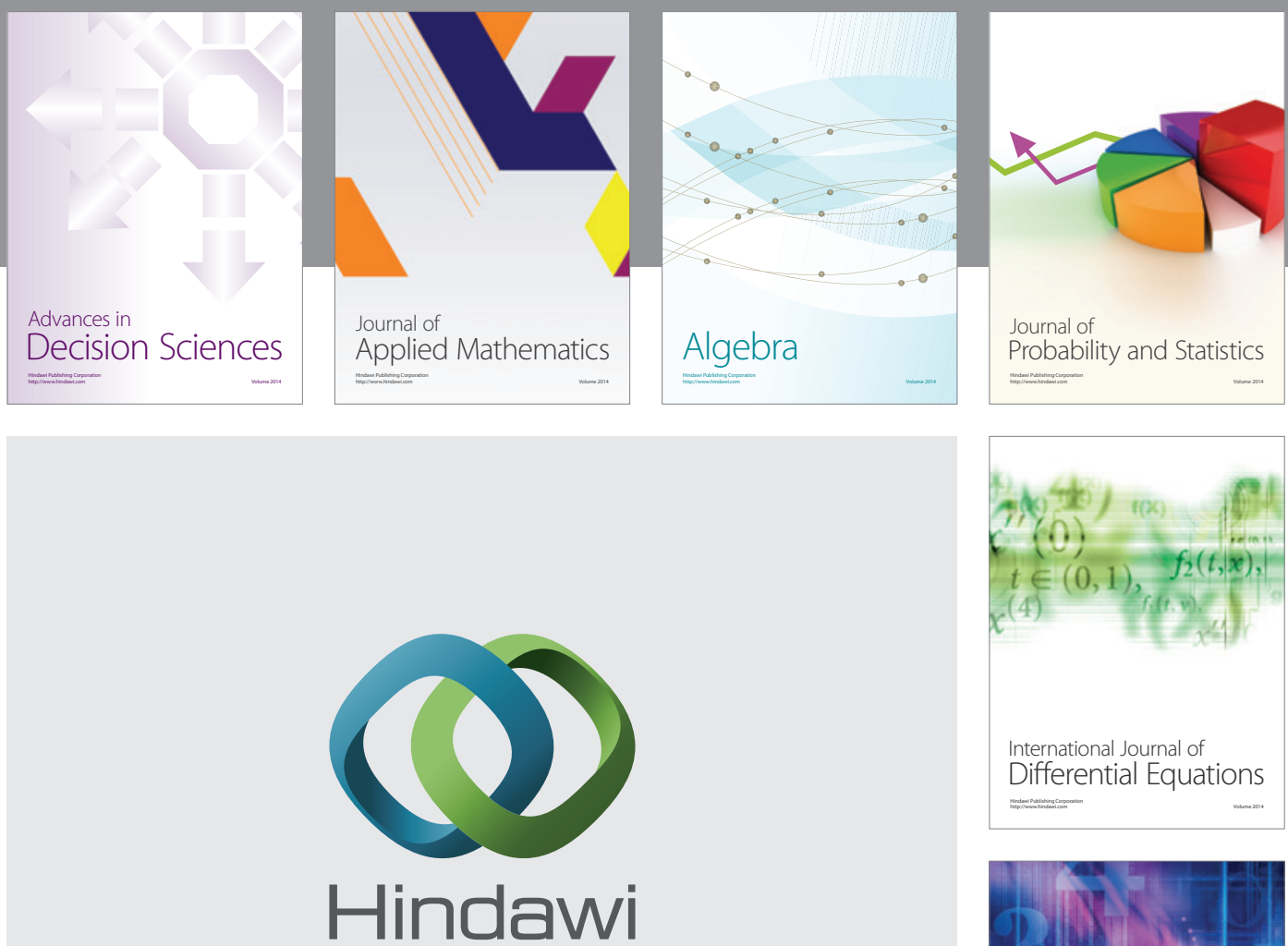

Submit your manuscripts at http://www.hindawi.com
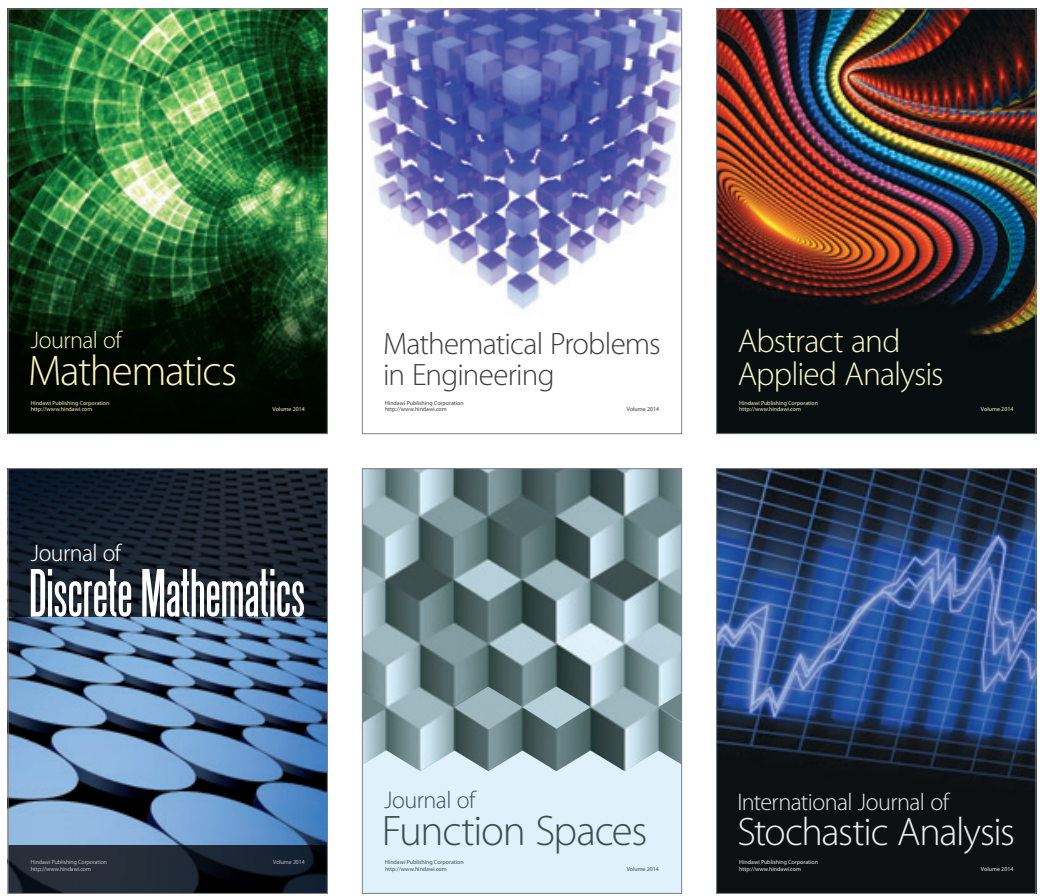

Journal of

Function Spaces

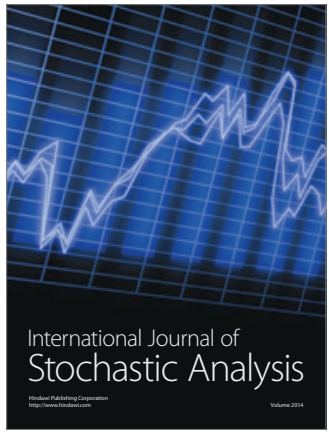

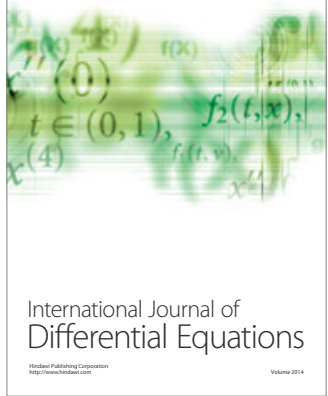
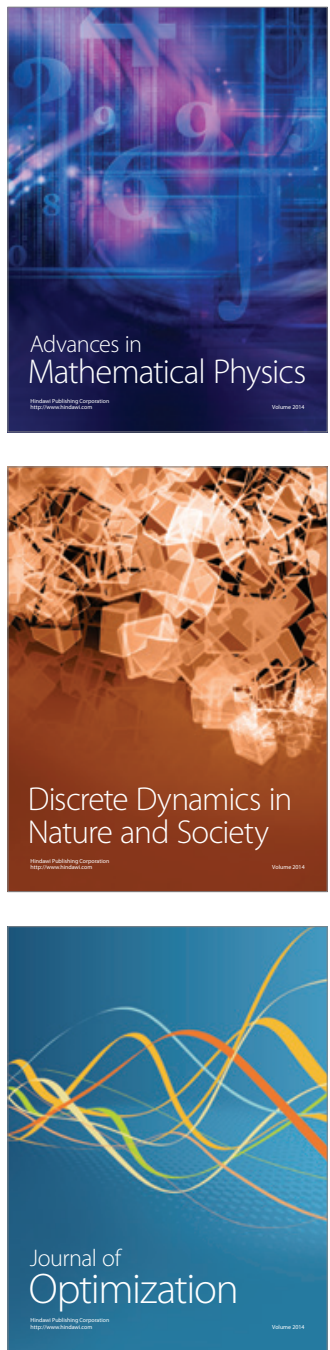